\title{
Dönel yaylar ile mesnetlenmiş bir karbon nanotüpün yerel olmayan Timoshenko kiriş teorisine göre serbest titreşim analizi
}

\author{
Mustafa Özgür YAYLI* \\ Uludă̆ Üniversitesi Müh. Fak. Inşaat Müh. Böl., Görükle kampüsü, Bursa \\ Geliș Tarihi (Recived Date): 24.11.2017 \\ Kabul Tarihi (Accepted Date): 14.09.2018
}

\begin{abstract}
Özet
Bu çalışmada dönel yaylar ile mesnetlenmiş bir karbon nanotüpün değişik sınır şartları için çözümü incelenmiştir. Küçük boyut etkisini problemin çözümüne dahil etmek için yerel olmayan elastisite teorisi kullanılmış ve Timoshenko kiriş teorisinde uygulanan kabuller göz önünde tutulmuştur. Yatay yer değiştirme fonksiyonu olarak Fourier sinüs serisi seçilmiştir. Sınır koşullarında esneklik sağlanması bakımından bir matematiksel dönüşüm olarak adlandırılan Stoke dönüşümü yüksek mertebeden sınır koşullarına uygulanmuştır. Matematiksel hesaplardan sonra içinde yay sabitleri ve boyut parametresi de bulunan bir katsaylar matrisi elde edilmiştir. Bu katsayılar matrisinin öz değerleri serbest tireşim frekanslarını vermektedir. Söz konusu katsayılar matrisinin doğal titreşim frekanslarını bulabilme yeteneği bir çok matematiksel örnekte test edilmiş ve literatürde bulunan sonuçlar ile karşılaştırılmıştır. Çözülen örneklerden titreşim frekanslarının dönel yay sabitlerine ve yerel olmayan parametreye bağll olarak değiştiği sonucuna ulaşılmıştır.
\end{abstract}

Anahtar kelimeler: Karbon nanotüp; dönel yay; serbest titreşim; Stoke dönüşümü, Fourier sinüs serisi.

\section{Free vibration analysis of a rotationally restrained carbon nanotube via nonlocal Timoshenko beam theory}

\begin{abstract}
In this study an investigation has been performed for a carbon nanotube with rotationally restrained boundary conditions. In order to examine the small size effect, nonlocal elasticity with Timoshenko beam assumptions have been used to solve the problem. Lateral deflection function has been chosen as a Fourier sine series. Stoke
\end{abstract}

\footnotetext{
*Mustafa Özgür YAYLI, ozguryayli@uludag.edu.tr, http://orcid.org/0000-0003-3778-3232
} 
transformation has been applied to the higher order boundary conditions. A coefficient matrix including nonlocal and spring parameters is derived after some mathematical calculations. The eigen values of this matrix gives the free vibration frequencies. The capabilities of present coefficient matrix has been tested through several numerical examples and compared the results avaliable in the literature. From the solved samples, the vibration frequencies have been reached as a result of the change depending on the rotational spring constants and the non-local parameter.

Keywords: Carbon nanotube, rotational restraints, free vibration, Stoke transformation, Fourier sine series.

\section{Giriş}

Yapı ve makine sistemlerinin boyutları mikro veya nano ölçekte olduğunda; klasik elastisite teorisi gerekli olan statik ve dinamik analizler için yetersiz kalmaktadır. Boyut etkisinin göz önüne alınmak zorunda olduğu nano veya mikro ölçekli yapılarda daha hassas sonuçlar elde etmek için mutlaka yüksek mertebeden elastisite teorileri kullanmak gereklidir. Son yıllarda yüksek mertebeden elastisite teorileri küçük boyuttaki makina veya yapı parçalarının analizinde sıklıkla kullanılmaktadır. Bu teorilerden bazıları (yerel olmayan elastisite teorisi) yapıyı zayıflatıcı etkilere sahipken bazıları ise yapıyı güçlendirici özelliklere sahiptir (değiştirilmiş gerilme çifti teorisi, gradyan elastisite teorisi). Literatürde bulunan ve araştırmacılar ilgisini çeken mikro ve makro boyutta ortaya çıkan farklılıklar günümüzde yoğun bir şekilde incelenmektedir. Chong ve Lam [1] epoksinin büyük ölçüde boyuta bağl1 olduğunu gözlemlemiştir. McFarland ve Colton [2] nano ölçekte yaptığı çalışmalarda; klasik elastisite teorisi kullanmanın uygun olmadığı üzerinde durmuştur.

Öncelikli olarak nano boyuttaki malzemeler ile deney yapmak oldukça pahalı ve son derece zahmetlidir. Dolayısıyla teorik çalışmalar büyük önem arz etmektedir. Teorik çalışmaların büyük çoğunluğu elbetteki deneysel çalışmaların yerini tutamaz ama büyük maliyetler gerektiren deneysel çalışmalara 1 şık tutabilir. Uygulamalı matematiği ve yüksek mertebeden elastisite teorilerini içeren bir çok teorik çalışma literatürde mevcuttur. Bu çalışmalar üç ana bölümde kategorilendirilebilir. Bunlar hibrit (atomiksürekli) [3-5], sürekli [6-8] ve atomik [9,10] ortam mekanikleridir. Klasik elastisite teorisi çok fazla hesaplama zahmeti gerektirmemekle birlikte hibrit ve atomik yöntemler ile büyük ölçüde benzerlik taşımaktadır [11,12]. Son yılllarda bir çok yüksek mertebeden elastisite teorileri ön plana çıkmıştır bunlar gerilme çifti teorisi $[13,14]$, yerel olmayan elastisite [15], yüzey elastisite [16] ve gradyan elastisite teorileridir [17]. Yang ve ark. [18] değiştirilmiş gerilme çifti teorisini geliştirmiş̧tir. Bu teoriden sonra değiştirilmiş gerilme çifti elastisite teorisi ve gradyan elastisite teorileriyle; nano ve micro ölçekteki yapıların statik dinamik ve stabilite analizleri değişik araştırmacılar tarafından gerçekleştirilmişstir [19-25].

Literatür taramasından görüleceği gibi çalışmaların çoğunda sınır koşulları rijit olarak alınmıştır. Bilindiği gibi rijit sınır koşulları sadece özel durumlarda söz konusudur ve uygulama aşamasında sınır koşullarının rijit olması çoğu zaman mümkün değildir. Hareket edebilir sınır koşullarıyla ilgili özellikle nano ölçekli yapılarda yapılan bilimsel çalışma sayısı oldukça azdır. Bu çalışmada Fourier serileri ve Stoke dönüşümü kullanılarak deforme olabilir sınır koşullarında nano ölçekteki bir Timoshenko kirişinin 
yerel olmayan elastisite teorisine göre titreşim analizi yapılmıştır. İlerleyen bölümlerde deforme olabilir sınır koşullarını da kapsayan bir katsayılar matrisi elde edilmiştir. Bu katsayılar matrisinin öz değerleri titreşim frekanslarını vermektedir. Çalışma kapsamında elde edilmiş olan katsayılar matrisi özellikle sınır koşullarının değişiminde esneklik sağlamaktadır. Ayrıca literatürde sıklıkla bulunan rijit sınır koşullarına ait çözümler hesaplanacak determinantta bulunan yay sabitlerine özel değerler verilerek elde edilebilmektedir.

\section{Teorik alt yapı}

\subsection{Problemin formülasyonu}

Şekil 1'de gösterilmiş olan basit ve dönel yaylar ile mesnetlenmiş bir Timoshenko kirişini göz önüne alalım. Bu çalışmada boyut etkisini göstermek için Eringen [15] tarafından öne sürülen yerel olmayan elastisite teorisi kullanılacaktır. Yerel olmayan elastisite ve Timoshenko kiriş teorisine göre hareket denklemleri aşağıda verilmiştir [26].

$$
\begin{aligned}
& E I \frac{d^{2} \phi}{d x^{2}}-K_{s} G A\left(\phi+\frac{d y}{d x}\right)+I \rho \omega^{2} \phi-\gamma^{2}\left(\rho A \omega^{2} \frac{d y}{d x}+I \rho \omega^{2} \frac{d^{2} \phi}{d x^{2}}\right)=0 \\
& K_{s} G A\left(\frac{d \phi}{d x}+\frac{d^{2} y}{d x^{2}}\right)+A \rho \omega^{2} y=0
\end{aligned}
$$

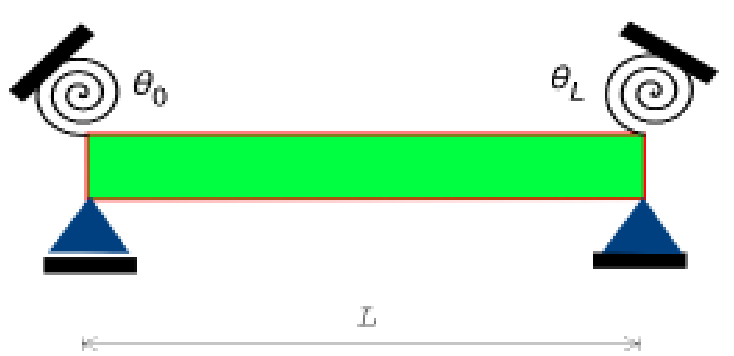

Şekil 1. Basit ve dönel yaylar ile mesnetlenmiş bir Timoskenko nano kirişi.

burada; y düşey doğrultaki yer değiştirmedir. $\varphi$ enkesitin eğilmeden kaynaklanan eğimini temsil etmektedir. EI ise eğilme rijitliğini göstermektedir. $G$ kayma modülü, A enkesit alanı, $\omega$ açısal frekans, I atalet momenti, E elastisite modülü ve $\rho$ kütle yoğunluğudur. $\mathrm{K}_{\mathrm{s}}$ terimi ise Timoshenko kiriş teorisinde kullanılan düzeltme katsayısıdır ve doğrudan enkesit geometrisiyle ilgilidir. (1) denkleminin x'e göre türevi alınırsa;

$$
E I \frac{d^{3} \phi}{d x^{3}}-K_{s} G A\left(\frac{d \phi}{d x}+\frac{d^{2} y}{d x^{2}}\right)+I \rho \omega^{2} \frac{d \phi}{d x}-\gamma^{2}\left(\rho A \omega^{2} \frac{d^{2} y}{d x^{2}}+I \rho \omega^{2} \frac{d^{3} \phi}{d x^{3}}\right)=0
$$

bulunur. Bu çalışmada herhangi bir titreşim modu için yatay yer değiştirme fonksiyonu ve eğim ifadesi aşağıdaki iki formülasyonla gösterilmiştir. 
$y(x)=\left\{\begin{array}{c}\delta_{0} \quad x=0 \\ \delta_{L} \quad x=L \\ \sum_{n=1}^{\infty} C_{n} \sin \left(\frac{n \pi x}{L}\right) \quad 0<x<L\end{array}\right\}$.

$\phi(x)=\left\{\begin{array}{c}\vartheta_{0} \quad x=0 \\ \vartheta_{L} \quad x=L \\ \sum_{n=1}^{\infty} D_{n} \cos \left(\frac{n \pi x}{L}\right) \quad 0<x<L\end{array}\right\}$.

burada L karbon nanotüpün boyudur. (4) ve (5) denklemlerindeki Fourier sabitleri aşağıdaki gibi yazılabilir.

$$
\begin{aligned}
& C_{n}=\frac{2}{L} \int_{0}^{L} y(x) \sin \left(\frac{n \pi x}{L}\right) d x, \\
& D_{n}=\frac{2}{L} \int_{0}^{L} \varphi(x) \cos \left(\frac{n \pi x}{L}\right) d x,
\end{aligned}
$$

\subsection{Stoke dönüsümü}

$\mathrm{Bu}$ alt bölümde; öncelikli olarak Stoke dönüşümünün nasıl gerçekleştirildiği hakkında teorik alt yap1 gösterilecektir [27-30]. Timoshenko kiriş teorisinin yerel olmayan elastisitede hareket denklemini çözmek için bir çok çalışma mevcuttur. Ancak hareketli sınır koşullarını problemin çözümünde göstermek için bu çalışmada Stoke dönüşümü kullanılmıştır. (4) denklemi bir kez türetilirse;

$$
y^{\prime}(x)=\sum_{n=1}^{\infty} \alpha_{n} C_{n} \cos \left(\alpha_{n} x\right)
$$

bulunur. Burada $\alpha_{n}$ katsayısı aşağıdaki formdadır:

$$
\alpha_{n}=\frac{n \pi}{L},
$$

(8) denklemi Fourier kosinüs serisi gibi gösterilebilir.

$$
y^{\prime}(x)=\frac{f_{0}}{L}+\sum_{n=1}^{\infty} f_{n} \cos \left(\alpha_{n} x\right),
$$

(10) denklemindeki katsayılar aşağıda verilmiştir.

$$
f_{0}=\frac{2}{L} \int_{0}^{L} y^{\prime}(x) d x=\frac{2}{L}[y(L)-y(0)],
$$


$f_{n}=\frac{2}{L} \int_{0}^{L} y^{\prime}(x) \cos \left(\alpha_{n} x\right) d x \quad(n=1,2 \ldots)$

Kısmi integrasyon uygulayarak aşağıdaki ifadeler elde edilebilir.

$$
\begin{aligned}
& f_{n}=\frac{2}{L}\left[y(x) \cos \left(\alpha_{n} x\right)\right]_{0}^{L}+\frac{2}{L}\left[\alpha_{n} \int_{0}^{L} y(x) \sin \left(\alpha_{n} x\right) d x\right], \\
& f_{n}=\frac{2}{L}\left[(-1)^{n} y(L)-y(0)\right]+\alpha_{n} C_{n},
\end{aligned}
$$

ve sonuç olarak birinci türev sınır koşulları ayrıklaştırılmış halde aşağıdaki gibi bulunur.

$$
\frac{d y(x)}{d x}=\frac{\delta_{L}-\delta_{0}}{L}+\sum_{n=1}^{\infty} \cos \left(\alpha_{n} x\right)\left(\frac{2\left((-1)^{n} \delta_{L}-\delta_{0}\right)}{L}+\alpha_{n} C_{n}\right)
$$

Yukarıda özetlenmiş olan matematiksel yöntem Stoke dönüşümü olarak ifade edilir. Benzer şekilde yüksek mertebeden türevler Stoke dönüşümü uygulanarak aşağıdaki gibi kolaylıkla bulunabilir.

$$
\begin{aligned}
& \frac{d^{2} y(x)}{d x^{2}}=-\sum_{n=1}^{\infty} \alpha_{n} \sin \left(\alpha_{n} x\right)\left(\frac{2\left((-1)^{k} \delta_{L}-\delta_{0}\right)}{L}+\alpha_{n} C_{n}\right) \\
& \frac{d^{3} y(x)}{d x^{3}}=\frac{\delta_{L}{ }^{\prime \prime}-\delta_{0} "}{L}+\sum_{n=1}^{\infty} \cos \left(\alpha_{n} x\right)\left(\frac{2\left((-1)^{n} \delta_{L} "-\delta_{0} "\right)}{L}-\alpha_{n}^{2}\left(\frac{2\left((-1)^{n} \delta_{L}-\delta_{0}\right)}{L}+\alpha_{n} C_{n}\right)\right) \\
& \frac{d^{4} y(x)}{d x^{4}}=-\sum_{n=1}^{\infty} \alpha_{n} \sin \left(\alpha_{n} x\right)\left(\frac{2\left((-1)^{n} \delta_{L} "-\delta_{0} "\right)}{L}-\alpha_{n}^{2}\left(\frac{2\left((-1)^{n} \delta_{L}-\delta_{0}\right)}{L}+\alpha_{n} C_{n}\right)\right)
\end{aligned}
$$

Benzer şekilde Stoke dönüşümü uygulanarak; (5) denklemiyle verilmiş olan Fourier kosinüs serisi de sınırlar için ayrıklaştırılabilir.

$$
\begin{aligned}
& \frac{d \phi(x)}{d x}=-\sum_{n=1}^{\infty} \alpha_{n} D_{n} \sin \left(\alpha_{n} x\right) \\
& \frac{d^{2} \phi(x)}{d x^{2}}=\frac{\vartheta_{L}^{\prime}-\vartheta_{0}^{\prime}}{L}-\sum_{n=1}^{\infty} \alpha_{n} \cos \left(\alpha_{n} x\right)\left(\frac{2\left((-1)^{n} \vartheta_{L}^{\prime}-\vartheta_{0}^{\prime}\right)}{L}-\alpha_{n}^{2} D_{n}\right), \\
& \frac{d^{3} \phi(x)}{d x^{3}}=\sum_{n=1}^{\infty} \alpha_{n} \sin \left(\alpha_{n} x\right)\left(\frac{2\left(\vartheta_{0}^{\prime}-(-1)^{n} \vartheta_{L}^{\prime}\right)}{L}+\alpha_{n}^{2} D_{n}\right)
\end{aligned}
$$

\subsection{Fourier katsayılarının bulunması}

(16), (17), (18), (19), (20) ve (21) denklemleri (2) ve (3) denklemlerinde yerlerine yazılırsa, $C_{n}, D_{n}$ katsayıları aşağıdaki gibi bulunur. 


$$
\begin{aligned}
& C_{n}=\frac{2 G K_{s} \alpha_{n} \Gamma_{1}\left(\vartheta_{0}^{\prime}-(-1)^{1+n} \vartheta_{L}^{\prime}\right)}{L\left(G K_{s}\left(-A \rho \omega^{2}+\alpha_{n}^{4} \Gamma_{1}\right)+\rho \omega^{2}\left(I \rho \omega^{2}+\alpha_{n}^{2} \Gamma_{2}\right)\right)} \\
& D_{n}=\frac{2\left(\rho \omega^{2}-G K_{s} \alpha_{n}^{2}\right) \Gamma_{1}\left(\vartheta_{0}^{\prime}+(-1)^{1+n} \vartheta_{L}^{\prime}\right)}{L \rho \omega^{2}\left(I \rho \omega^{2}-\alpha_{n}^{2} \Gamma_{1}\right)+G L K_{s}\left(\alpha_{n}^{4} \Gamma_{1}-\rho \omega^{2} \Gamma_{3}\right)}
\end{aligned}
$$

burada;

$$
\begin{aligned}
& \Gamma_{1}=E I-\gamma^{2} I \rho \omega^{2} \\
& \Gamma_{2}=-E I+\gamma^{2} I \rho \omega^{2}-G\left(A \gamma^{2}+I\right) K_{s} \\
& \Gamma_{3}=A+\left(A \gamma^{2}+I\right) \alpha_{n}^{2}
\end{aligned}
$$

şeklinde ifade edilmiştir.

\subsection{Timoshenko kiriş teorisinde yerel olmayan sınır koşulları}

Şekil 1' deki Timoshenko kiriş teorisinde yerel olmayan sınır koşulları [26] dönel yayları da içerecek şekilde aşağıdaki gibi yeniden düzenlenebilir.

$$
\begin{aligned}
& \theta_{0} \frac{d \varphi(x)}{d x}=E I \vartheta_{0}^{\prime}-\gamma^{2}\left(\rho A \omega^{2} y+\rho I \omega^{2} \vartheta_{0}^{\prime}\right), \\
& y=0, \quad x=0, \\
& \theta_{L} \frac{d \varphi(x)}{d x}=E I \vartheta_{L}^{\prime}-\gamma^{2}\left(\rho A \omega^{2} y+\rho I \omega^{2} \vartheta_{L}^{\prime}\right), \\
& y=0, \quad x=L,
\end{aligned}
$$

burada $\theta_{0}$ ve $\theta_{L}$ sirasiyla sinırlardaki dönel yay sabitleridir. (19), (13), (22), (23) ve (17) denklemleri (27)-(30) denklemlerinde yerlerine yazılırsa aşağıdaki iki denklem takımı bulunur.

$$
\begin{aligned}
& \left(-\Lambda_{1}+\sum_{n=1}^{\infty} \frac{2 L \theta_{0} \Lambda_{1} \Lambda_{2}}{\Lambda_{3}+\Lambda_{4}}\right) \vartheta_{0}^{\prime}+\left(\sum_{n=1}^{\infty} \frac{2 L \theta_{0} \Lambda_{1} \Lambda_{2}(-1)^{1+n}}{\Lambda_{3}+\Lambda_{4}}\right) \vartheta_{L}^{\prime}=0 \\
& \left(\sum_{n=1}^{\infty} \frac{2 L \theta_{L} \Lambda_{1} \Lambda_{2}(-1)^{1+n}}{\Lambda_{3}+\Lambda_{4}}\right) \vartheta_{0}^{\prime}+\left(-\Lambda_{1}+\sum_{n=1}^{\infty} \frac{2 L \theta_{L} \Lambda_{1} \Lambda_{2}}{\Lambda_{3}+\Lambda_{4}}\right) \vartheta_{L}^{\prime}=0
\end{aligned}
$$

burada;

$$
\begin{aligned}
& \Lambda_{1}=E I-\gamma^{2} I \rho \omega^{2} \\
& \Lambda_{2}=L^{2} \rho \omega^{2}-G n^{2} \pi^{2} K_{S}
\end{aligned}
$$


$\Lambda_{3}=L^{2} \rho \omega^{2}\left(-\operatorname{EIn}^{2} \pi^{2}+\left(L^{2}+n^{2} \pi^{2} \gamma^{2}\right) I \rho \omega^{2}\right)$

$$
\Lambda_{4}=G\left(B n^{4} \pi^{4}-\left(L^{2}+n^{2} \pi^{2} \gamma^{2}\right) \times\left(A L^{2}+n^{2} \pi^{2} I\right) \rho \omega^{2}\right) K_{s}
$$

şeklinde gösterilmiştir. (31) ve (32) denklemleri matris formunda aşağıdaki gibi gösterilir.

$\left[\begin{array}{ll}\chi_{11} & \chi_{12} \\ \chi_{21} & \chi_{22}\end{array}\right]\left[\begin{array}{l}\vartheta_{0}^{\prime} \\ \vartheta_{L}^{\prime}\end{array}\right]=0$,

burada;

$$
\begin{aligned}
& \chi_{11}=-\Lambda_{1}+\sum_{n=1}^{\infty} \frac{2 L \theta_{0} \Lambda_{1} \Lambda_{2}}{\Lambda_{3}+\Lambda_{4}} \\
& \chi_{12}=\sum_{n=1}^{\infty} \frac{2 L \theta_{0} \Lambda_{1} \Lambda_{2}(-1)^{1+n}}{\Lambda_{3}+\Lambda_{4}}, \\
& \chi_{21}=\sum_{n=1}^{\infty} \frac{2 L \theta_{L} \Lambda_{1} \Lambda_{2}(-1)^{1+n}}{\Lambda_{3}+\Lambda_{4}}, \\
& \chi_{22}=-\Lambda_{1}+\sum_{n=1}^{\infty} \frac{2 L \theta_{L} \Lambda_{1} \Lambda_{2}}{\Lambda_{3}+\Lambda_{4}},
\end{aligned}
$$

şeklinde ifade edilmiştir. (37) denklemindeki katsayılar matrisinin özdeğerleri determinantın sıfıra eşitlenmesiyle bulunur;

$\operatorname{det}\left|\chi_{i j}\right|=0 \quad(i, j=1,2)$.

(42) denkleminden elde edilecek karakteristik denklemin kökleri $\theta_{0}$ ve $\theta_{L}$ parametrelerinin çeşitli değerleri için bulunabilir. Bulunacak özdeğerler Timoshenko kiriş teorisine göre yerel olmayan kirişlerin serbest titreşim frekansları olacaktır. $\theta_{0}$ ve $\theta_{L}$ parametrelerine sonsuz büyük değerler verilirse ankastre mesnetli nanotüplerin frekansları bulunur. $\theta_{0}$ ve $\theta_{L}$ parametreleri sıfır olursa basit mesnetli nanotüplerin frekansları elde edilir. Görüldüğü gibi kurulan formülasyonla çeşitli sınır şartlarındaki nanotüplerin frekansları kolaylıkla bulunabilmektedir.

\section{Sayısal sonuçlar}

$\mathrm{Bu}$ bölümde önceki kısımlarda elde edilmiş olan formülasyonlar uygulanarak çeşitli sonuçlar elde edilmiştir. Ayrıca dönel yay sabitlerine özel değerler vermek suretiyle klasik sınır koşulları için de sonuçlar elde edilebilir. Örnek olarak yay sabitleri $\left(\theta_{0}=0, \theta_{L}=0\right)$ alınırsa, (42) denklemi basit_menetli_karbon nanotüplerin çözümü için kullanılabilir. Benzer şekilde yay sabitleri $\left(\overrightarrow{\theta_{0}}=\infty, \overline{\theta_{L}}=\infty\right)$ olarak yazılırsa; iki tarafı ankastre karbon nanotüpün açısal frekansları bulunabilir.

\subsection{Sayusal örnekler}

$\mathrm{Bu}$ alt bölümde çeşitli sayısal örnekler; kurulan formülasyonla çözülmüştür. Sayısal analizlerde $\mathrm{E}=1 \mathrm{TPa}, \mathrm{Ks}=9 / 10$ olarak alınmıştır. Literatürle uyumlu olarak, $\rho=2.3 \mathrm{~g} \mathrm{~cm}$

${ }^{3}$ alınmıştır [26]. Karbon nanotüpün çap ve uzunluk oranı d / L = 1/10 seçilmiştir. 
Çözülmüş olan örneklerin tamamında $\theta_{0}=\theta_{L}=0.01 \mathrm{nN} / \mathrm{nm}$ alınmıștır. Çeşitli yerel olmayan parametreler için çözümler bulunarak Şekil 2 'de gösterilmiştir. Bu örnekten yerel olmayan etkilerin yüksek modlarda daha ön plana çıktığı gözlenmiștir. Söz konusu sonuçlar literatür ile uyumludur.

(42) denkleminden görüleceği gibi determinantı oluşturan elemanlar sonsuz serilerden oluşmaktadır ve serilerde kullanılacak terim sayısının seçilmesi büyük önem arz etmektedir. İkinci örnekte çeşitli terim sayıları kullanılarak elde edilen sonuçlar Şekil 4-5' te şematik olararak sunulmuștur. Söz konusu sayısal çalışmada farklı terim sayılarıyla (42) denklemiyle verilmiş olan determinant hesaplanmış ve ilk iki mod için grafiksel olarak karşılaştırma yapılmıştır. Bu örnekte sabit yerel olmayan parametre değeri için düşey yer değiştirme fonksiyonları ilk iki mod için çizdirilmiş ve kullanılacak terim sayısı test edilmiştir. Şekil 4-5' ten görüldüğü gibi ilk 50 terimin kullanılması her iki mod için de yeterlidir ve yeterli hassasiyete sahiptir. Bu aşamadan sonra terim sayısının artırılması sadece hesaplama zahmeti getirecektir.

Son örnekte farklı yerel olmayan paramtrelerin ilk dört mod üzerindeki etkileri tartışılmıştır. İlk dört mod için düşey yer değiştirme fonksiyonları elde edilerek Şekil 6-9 da grafiksel olarak verilmiștir. Bu grafiklerden yüksek modlarda yerel olmayan etkilerin ön plana çıktığı ve belirginleștiği gözlenmiștir. Problemi klasik elastisite teorisine göre çözmenin özellikle nano ölçekteki yapılarda ne kadar yanlıș olduğu ortadadır. Problemi çözmek için küçük boyut etkisini göz önüne olan yüksek mertebeden elastisite teorilerinin kullanılmasının zorunlu olduğu söz konusu șekillerden ortaya çıkmaktadır. Özellikle yüksek modlarda klasik elastistisite teorisi oldukça hatalı sonuçlar vermektedir. Benzer şekilde farklı yay sabitleri için yerel olmayan etkilerin katkıları incelenmiştir ve literatürle uyumlu sonuçlar elde edilmiștir. Literatürden bilindiği gibi yerel olmayan etkilerin yüksek modlarda daha ön plana çıkmaktadır. Bu çalışmada yapılan çözümlerde de yerel olmayan parametrenin yüksek modlara etkisi daha fazladır. Aşağıdaki şekillerden görüldügü gibi terim sayısının belli bir değerinden sonra titreşim frekansları aynı değerde kalmaktadır. Terim sayısı için sonsuz bir değer alındığında çözümler kapalı olacaktır. Çözümlerin yapılması için Fortran programlama dilinde bir program yazılmış ve sayısal veriler problemde kullanılan parametrelerin çeşitli değerlerine göre elde edilmiştir.

Literatürde bulunan çalışmaları bir çoğunda sınır koşulları olarak ya basit mesnetli Timoshenko kirişleri kullanılmış veya rijit sınır koşullarına sahip çubuklar tercih edilmiştir [31,32]. Sınır koşullarının rijit seçilmesinin temel nedeni analitik çözüm için gerekli olan fonksiyonların kolay önerilebilir olmasıdır. Ancak gerçek fiziksel nesnelerde bulunan sınır koşulları hiç bir zaman rijit olmayacaktır. Bu çalışmada gerçek fiziksel bir problem çözmek için rijit olmayan sınır koşulları tercih edilmiştir.

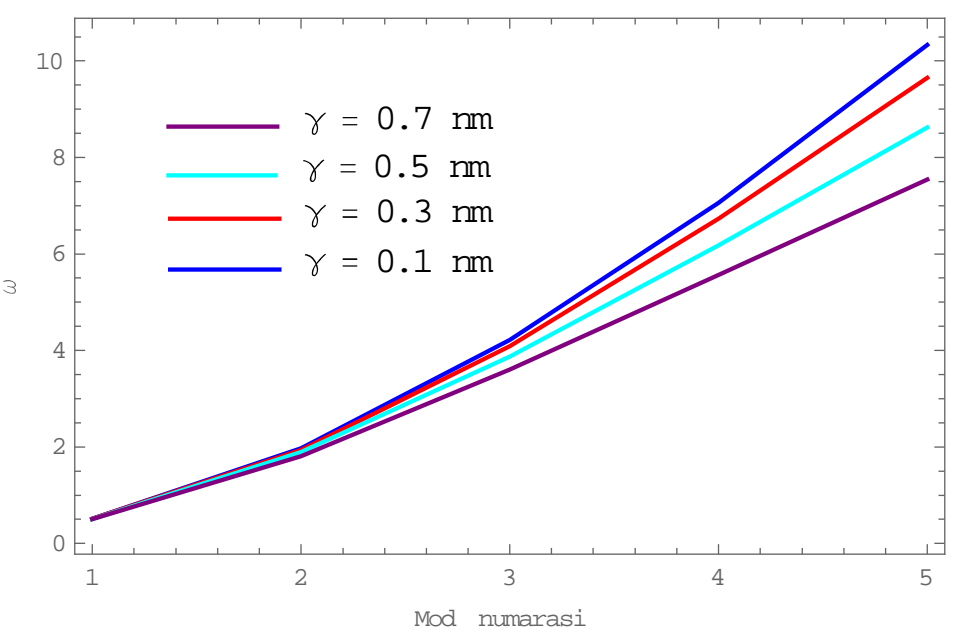

Şekil 2. Farklı modıara aıt açısaı trekansıarın tarkıı yereı oımayan parametre değerleri için değişimi. 


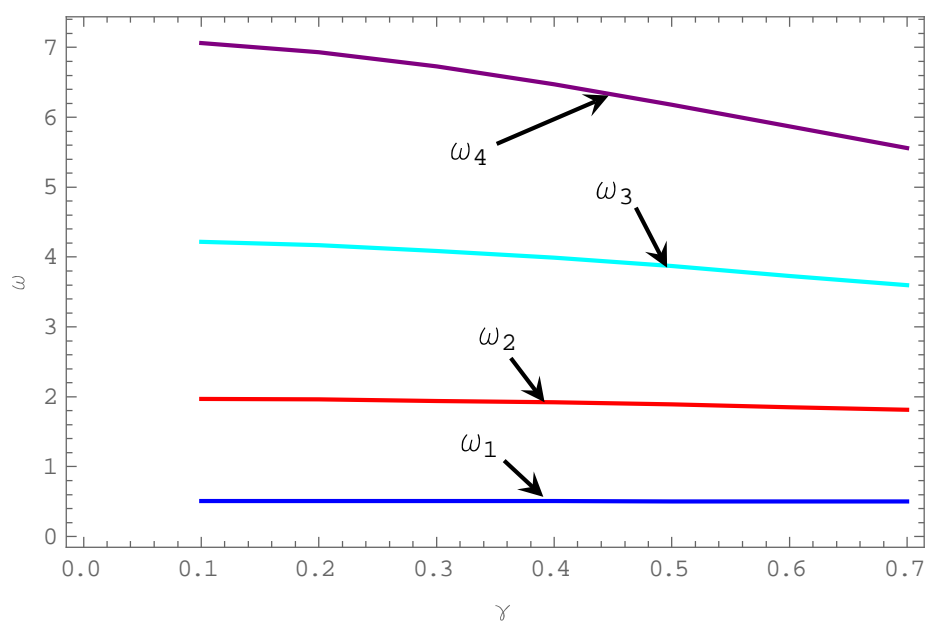

Şekil 3. İlk dört açısal frekansın yerel olmayan parametreye bağlı değişimi.

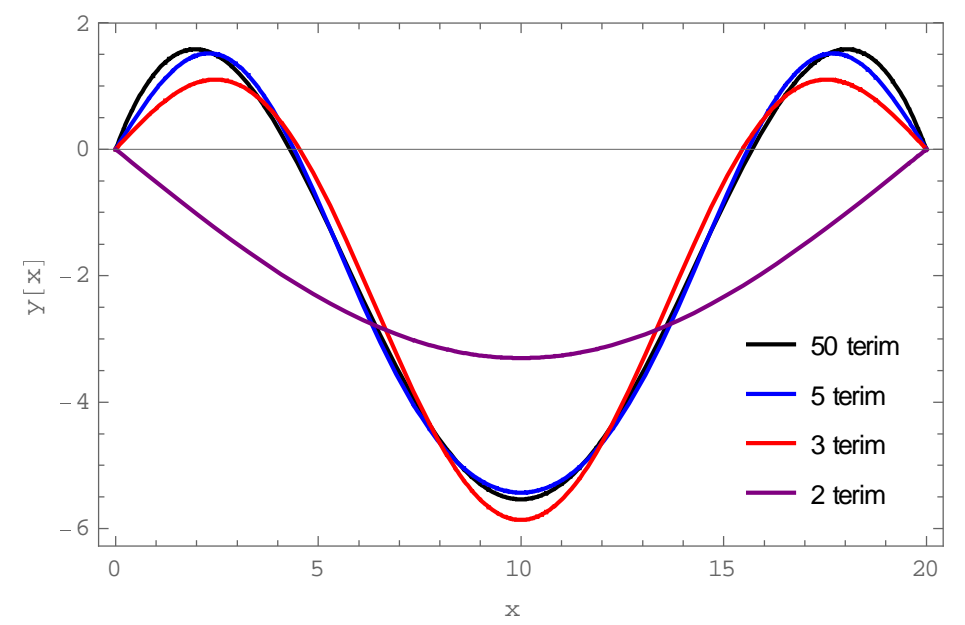

Şekil 4.a. Birinci mod için farklı terim sayılarıyla bulunmuş olan sonuçlar (2,3,5 ve 50 terim).

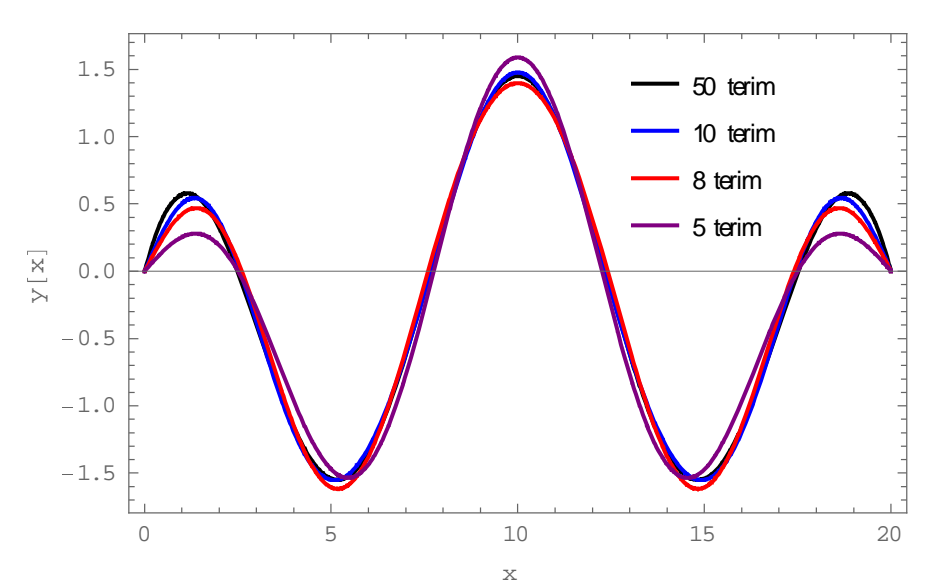

Şekil 4.b. Birinci mod için farklı terim sayılarıyla bulunmuş olan sonuçlar (5, 8, 10 ve 50 terim). 


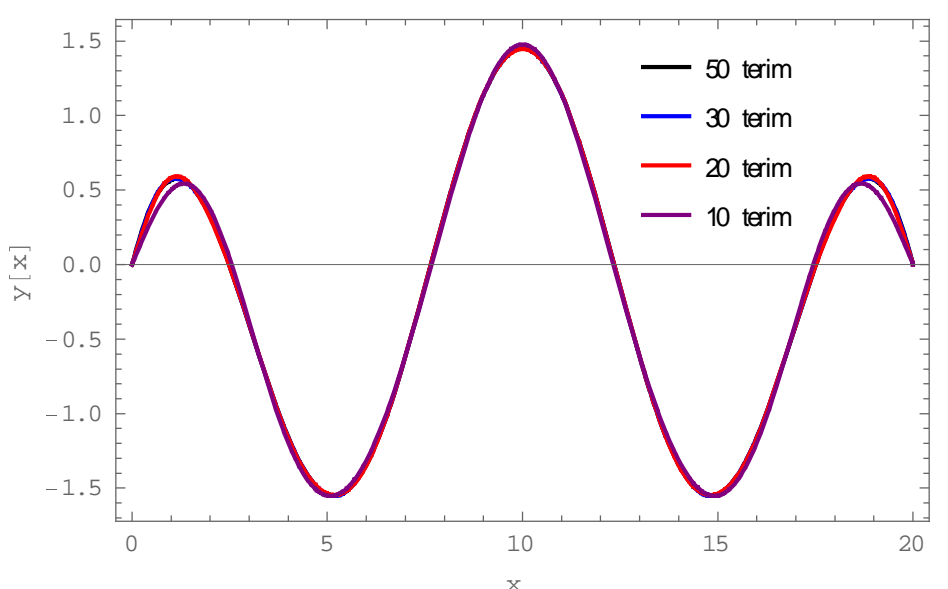

Şekil 4.c. Birinci mod için farklı terim sayılarıyla bulunmuş olan sonuçlar (10, 20, 30 ve 50 terim).

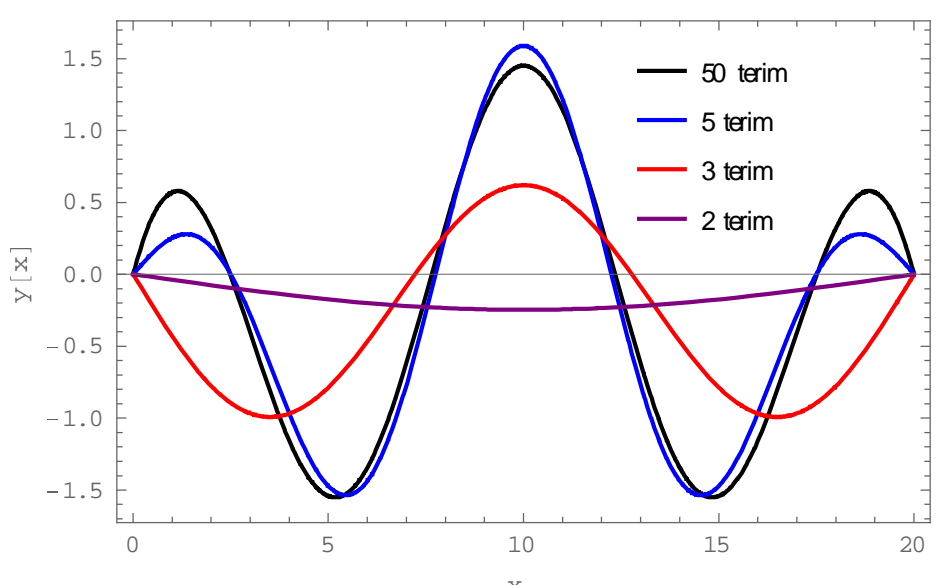

Şekil 5a. İkinci mod için farklı terim sayılarıyla bulunmuş olan sonuçlar (2,3,5 ve 50 terim).

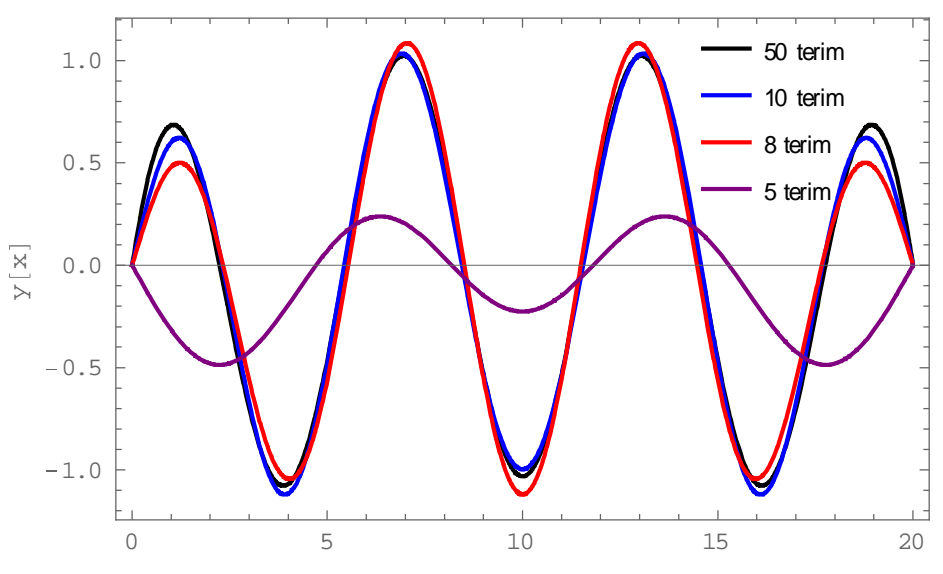

Şekil 5b. İkinci mod için farklı terim sayılarıyla bulunmuş olan sonuçlar $(5,8,10$ ve 50 terim). 


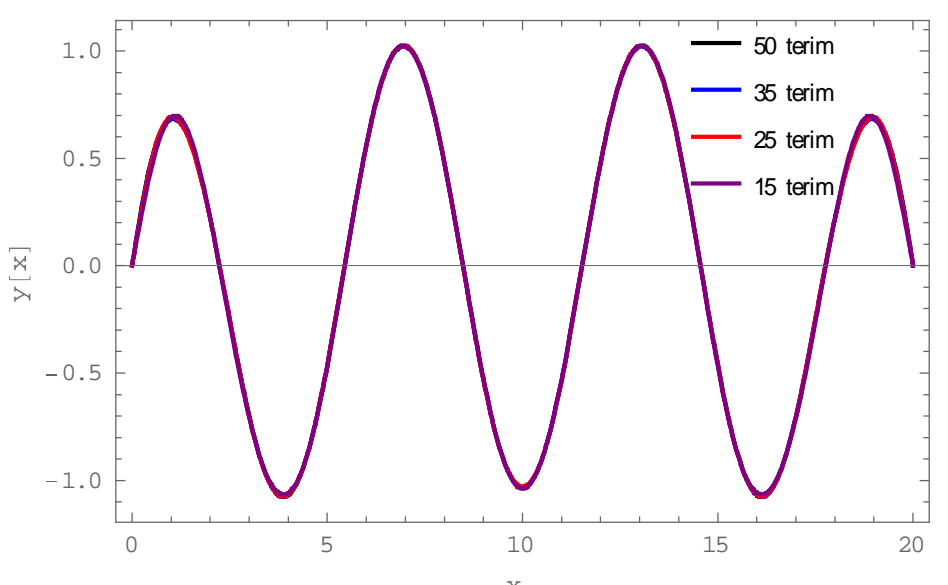

Şekil 5c. İkinci mod için farklı terim sayılarıyla bulunmuş olan sonuçlar $(15,25,35$ ve 50 terim).

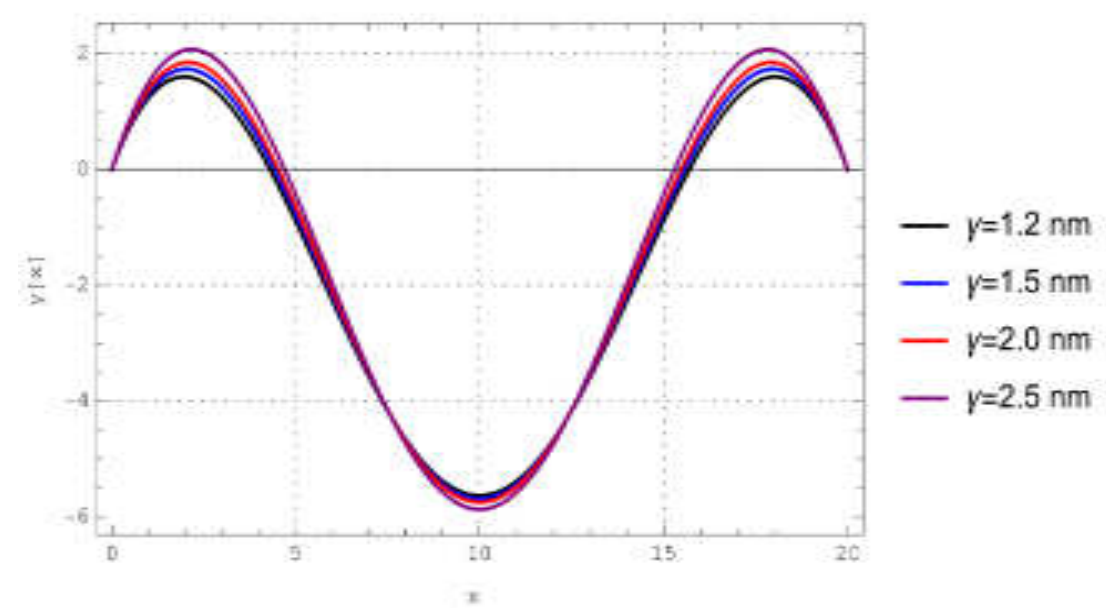

Şekil.6. Farklı yerel olmayan parametrelerin birinci moda etkisi.

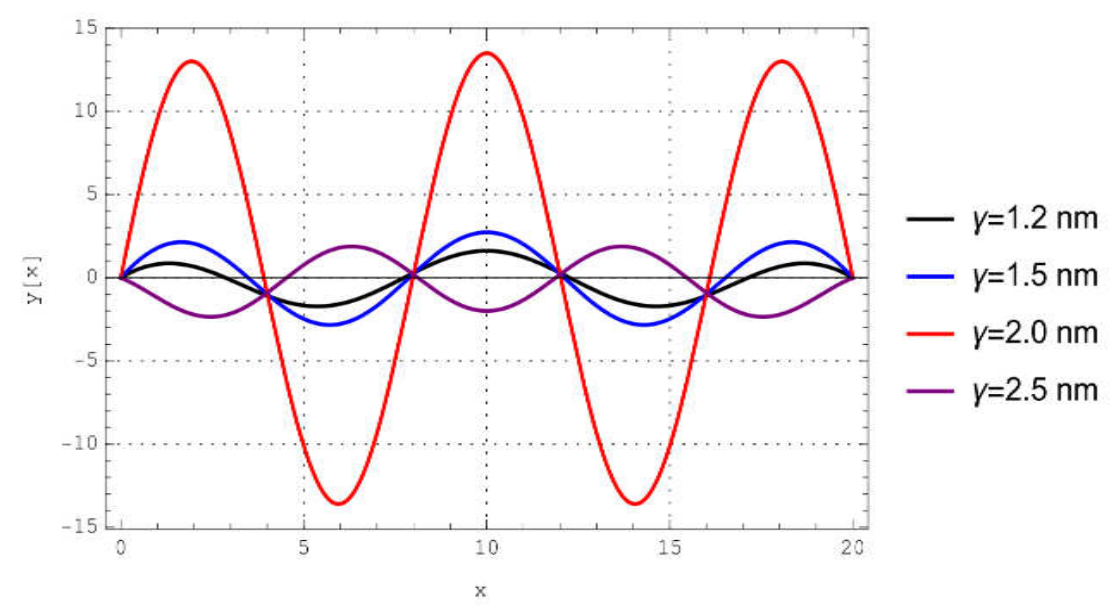

Şekil 7. Farklı yerel olmayan parametrelerin ikinci moda etkisi. 


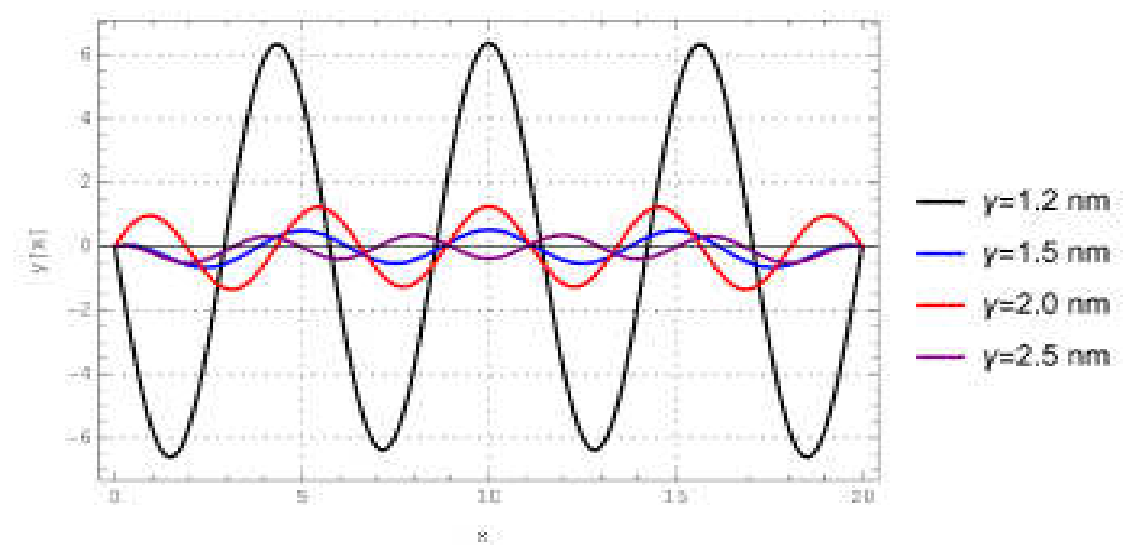

Şekil.8. Farklı yerel olmayan parametrelerin üçüncü moda etkisi.

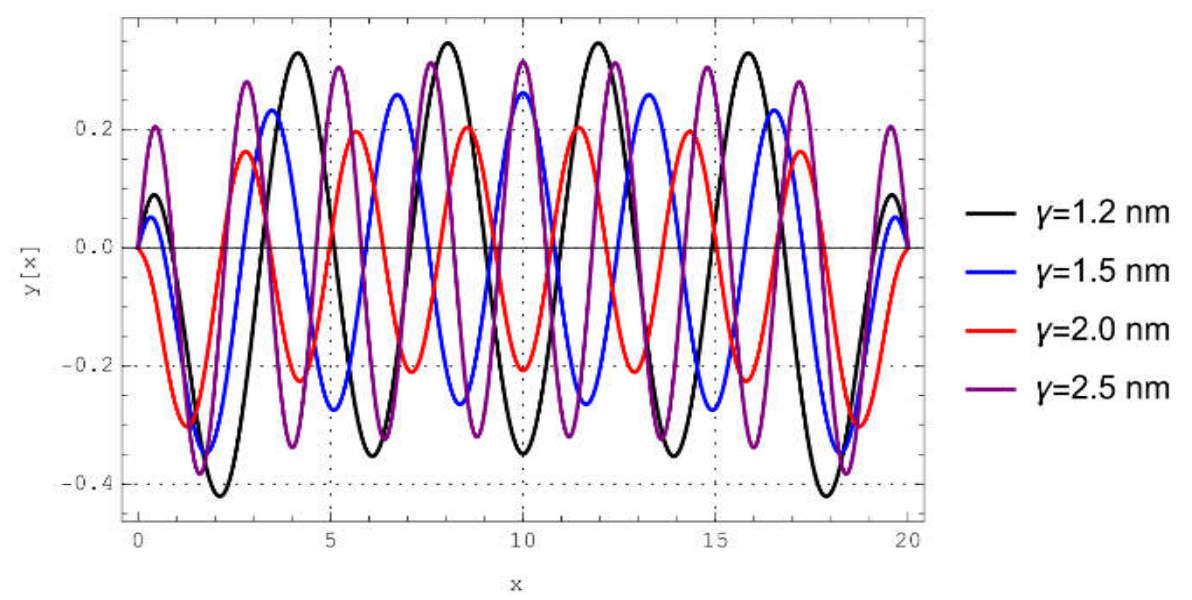

Şekil.9. Farklı yerel olmayan parametrelerin dördüncü moda etkisi.

\section{Sonuçlar}

Basit ve dönel yaylar ile mesnetlenmiş Timoshenko kirişlerinin yerel olmayan elastisite teorisine göre titreşim analizleri incelenmiştir. Yatay yer değiştirme ve eğim fonksiyonu olarak sırasuyla Fourier sinus ve kosinüs serileri seçilmiştir. Bu serilere Stoke dönüşümleri uygulanmış ve problemi yöneten denklemlerde yerlerine yazılmıştır. $\mathrm{Bu}$ şekilde Fourier katsayıları elde edilmiştir. Bulunmuş olan katsayılar yüksek mertebeden sınır koşullarında yerlerine yazılarak katsayılar matrisi elde edilmiştir. Bu katsayılar matrisinin öz değerleri titreşim frekanslarını vermektedir. Bulunan sonuçlar aşağıda sıralanmıştır:

- Bulunan katsayılar matrisi farklı sınır koşulları için titreşim frekanslarını vermektedir.

- Klasik yöntemlerde olduğu gibi her defasında yeni sınır koşulu yazılarak yeni denklem çözmeye ihtiyaç duyulmadan doğrudan sonuca gidilmektedir.

- Titreşim davranışının yay sabitlerine bağlı olduğu gösterilmiştir.

- Boyut etkisi sert yaylarda daha fazla ortaya çıkmaktadır.

- Bu metodla herhangi bir zamandaki yatay deplasman doğrudan bulunabilecektir

- Kullanılan terim sayısı arttıkça bulunan sonuçlar birbirine çok yaklaşmaktadır ve bu da kullanılan yöntemin doğruluğunu ispatlamaktadır. 
- Yüksek modlarda daha fazla terim kullanmanın gerekli olduğu şekiller ile gösterilmiştir.

\section{Kaynaklar}

[1] Chong, A.C.M., Lam, D C C., Strain gradient plasticity effect in indentation hardness of polymers, Journal of Materials Research, 14, 4103-4110, (1999).

[2] McFarland, A.W., Colton, J.S., Role of material microstructure in plate stiffness with relevance to micro cantilever sensors, Journal of Micromechanics Microengineering, 15, 1060-1067, (2005).

[3] Bodily, B.H., Sun, C.T., Structural and equivalent continuum properties of single-walled carbon nanotubes, International Journal of Materials and Product Technology, 18(4-6), 381-397, ( 2003).

[4] Li, C., Chou, T.W., A structural mechanics approach for the analysis of carbon nanotubes, International Journal of Solids and Structures, 40(10), 24872499, (2003) .

[5] Li, C., Chou, T.W., Single-walled carbon nanotubes as ultrahigh frequency nanomechanical resonators, Physical Review B, 68(7), 073405, (2003).

[6] Aydogdu, M., Axial vibration of the nanorods with the nonlocal continuum rod model, Physica E, 41, 5, 861-864, (2009).

[7] Pradhan, S.C., Phadikar, J. K., Small scale effect on vibration of embedded multilayered graphene sheets based on nonlocal continuum models, Physics Letters A, 373, 11, 1062- 1069, (2009).

[8] Reddy, J.N., Pang, S.D., Nonlocal continuum theories of beams for the analysis of carbon nanotubes, Journal of Applied Physics, 103, 2, 023511, (2008).

[9] Ball, P., Roll up for the revolution, Nature, 414, 6860, 142-144, (2001).

[10] Baughman, R.H., Zakhidov, A.A., de Heer, W.A., Carbon nanotubes-the route toward applications, Science, 297, 5582, 787-792, (2002).

[11] Wang, C.M., Tan, V.B C., Zhang, Y.Y., Timoshenko beam model for vibration analysis of multi-walled carbon nanotubes, Journal of Sound and Vibration, 294, 4, 1060-1072, (2006).

[12] Wang, Q., Vardan, V. K., Characteristics of carbon nanotubes, International Journal of Solids and Structures, 43, 254-265, (2005).

[13] Toupin, R.A., Elastic materials with couple-stresses, Archieve of Rational Mechanics and Analysis, 11, 385-414, (1962).

[14] Mindlin, R.D., Tiersten, H.F., Effects of couple-stresses in linear elasticity, Archieve of Rational Mechanics and Analysis, 11, 415-448, (1962).

[15] Eringen, A.C., Nonlocal polar elastic continua, International Journal of Engineering Science, 10, 1-16, (1972).

[16] Gurtin, M. E., Weissmuller, J. Larche, F., The general theory of curved deformable interfaces in solids at equilibrium, Philosophical Magazine, 78, 1093-1109, (1998).

[17] Aifantis, E.C., Strain gradient interpretation of size effects. International Journal of Fracture, 95, 1-4, (1999).

[18] Yang, F., Chong, A.C.M., Lam, D.C., Tong, P., Couple stress based strain gradient theory for elasticity. International Journal of Solids and Structures. 39 2731-2743, (2002). 
[19] Park, S.K., Gao, X.L., Bernoulli-Euler beam model based on a modified couple stress theory, Journal Micromechanics Microengineering 16, 2355-2359, (2006).

[20] Ma, H.M., Gao, X.L., Reddy, J.N., A microstructure-dependent Timoshenko beam model based on a modified couple stress theory, Journal of the Mechanics and Physics of Solids 56, 3379-3391, (2008).

[21] Simsek, M., Dynamic analysis of an embedded microbeam carrying a moving microparticle based on the modified couple stress theory. International Journal of Engineering and Science. 48, 1721-1732, (2010).

[22] Akgoz, B., Civalek, O., Strain gradient elasticity and modified couple stress models for buckling analysis of axially loaded micro-scaled beams. International Journal of Engineering Science. 49, 11, 1268-1280, (2011).

[23] Kahrobaiyan, M.H., Asghari, M., Rahaeifard, M., Ahmadian M. T., A nonlinear strain gradient beam formulation. International Journal of Engineering Science, 49, 1256-1267, (2011).

[24] Artan, R., Tepe, A., Nonlocal effects in curved single-walled carbon nanotubes, Mechanics of Advanced Materials and Structures, 18, 347-351, (2011).

[25] Xu, L.Z., Jia, X.L., Electromechanical dynamics for micro beams. International Journal of Structural Stability and Dynamics, 6(2), 233-251, (2006).

[26] Wang, C.M., Zhang, Y.Y., He, X.Q., Vibration of nonlocal Timoshenko beams. Nanotechnology.18, 10, 105401. (2007).

[27] Yayli, M.Ö., An analytical solution for free vibrations of a cantilever nanobeam with a spring mass system, Int J Eng Appl Sci, 7(4), 10-18, (2016).

[28] Yayli, M.Ö., Buckling analysis of a rotationally restrained single walled carbon nanotube.Acta Physica Polonica A. 127(3), 678-683, (2015).

[29] Yayli, M.Ö., A compact analytical method for vibration analysis of singlewalled carbon nanotubes with restrained boundary conditions, Journal of Vibration and Control. 22, 10, 2542-2555, (2016).

[30] Yayli, M.Ö., Buckling analysis of a cantilever single-walled carbon nanotube embedded in an elastic medium with an attached spring, Micro \& Nano Letters. 12, 4, 255-259, (2017) .

[31] Benzair, A., Tounsi, A., Besseghier, A., Heireche, H., Moulay, N., Boumia, L. The thermal effect on vibration of single-walled carbon nanotubes using nonlocal Timoshenko beam theory, Journal of Physics D: Applied Physics, 41(22), 225404 (2008).

[32] Ke, L.L., Yang, J., Kitipornchai, Nonlinear free vibration of functionally graded carbon nanotube-reinforced composite beams, Composite Structures, 92(3), 676-683, (2010). 